chain reaction for Mycobacterium tuberculosis and were stained for acid-fast bacilli.

Results We performed 33 procedures. Median age of children was 56.4 months (13-360). 10 children were diagnosed with pulmonary tuberculosis and another 1 with latent tuberculosis. Mean MOPS score was 4 (range 2-8). The degree of sedation achieved enabled all procedures to be completed without requiring additional drugs. The only side effect registered was transitory euphoria in 6 cases (18\%). Sedation was always well accepted by both parents and health workers.

Conclusions This study has shown that the combined use of intranasal Midazolam and Ketamine appears to be a safe and effective method to achieve children's short-term sedation and to facilitate the gastric lavage procedures.

\section{PAEDIATRIC STOOL TESTING PRACTICES IN IRELAND AND POTENTIAL INFLUENCE ON EPIDEMIOLOGY OF ROTAVIRUS GASTROENTERISIS (RVGE) AND ROTAVIRUS IMIMUNISATION}

doi:10.1136/archdischild-2012-302724.0866

${ }^{1} \mathrm{~F}$ Carroll, ${ }^{2} \mathrm{~J}$ Powell, ${ }^{2 \mathrm{R}}$ McMahon, ${ }^{3} \mathrm{D}$ O'Rourke, ${ }^{3} \mathrm{C}$ O'Donnell, ${ }^{2} \mathrm{~T}$ Dooly, ${ }^{2} \mathrm{~N}$ O'Connell, ${ }^{4} \mathrm{R}$ Fitzgerald, 'RK Philip. 'Paediatrics; 'Microbiology; ${ }^{3}$ Serology, University Hospital Limerick; 'Population Health, HSE West, Limerick, Ireland

Background and Aims Rotavirus vaccination has not been taken up in Ireland. We aimed to conduct a regional audit of gastroenteritis and paediatric stool testing patterns to generate better epidemiological data to support a rotavirus vaccination programme in Ireland.

Methods A retrospective audit of infants and children $<5 y$ rs with Hospital Inpatient Enquiry (HIPE) coded gastroenteritis admitted between 2005 and 2010. Stool testing patterns from the community and hospitals, from the Mid-West Ireland was analysed. Trends of Roatvirus, Adenovirus, Bacterial, Stool test Negative gastroenteritis was tabulated. Audit was approved by hospital audit committee.

Results The incidence of rotavirus in the tested paediatric population is $26 \%$ and that of adenovirus is $5 \%$. Rotavirus peaked in 2006 with $30 \%$ testing positive, compared with $22 \%$ in 2008 and $25 \%$ in 2010. The positive pick up rate for stool culture \& sensitivity (C\&S) by community clinicians and Hospital respecively was 4\%, 7\% and $9 \%$ in 2001, 2006 and 2010. While only 1640 stool samples were tested for rotavirus over 5 years, 7534 samples were tested for C\&S with the vast majority not warranting any anti-microbial treatment. In a $5 \mathrm{yr}$ period, GPs in community only requested rotavirus on 287 stool samples, while testing 3353 for C\&S.

Conclusion Our rotavirus epidemiological data, based on which the decision not to incorporate an oral rotavirus vaccine to the National schedule, is possibly inaccurate reflecting our stool testing practices. Further reduction of clinical burden due to RVGE could be achieved by incorporating rotavirus immunization to the National programme.

\section{EPIDEMIOLOGY OF BACTERIAL MENINGITIS IN TUNISIAN CHILDREN (2000-2011)}

doi:10.1136/archdischild-2012-302724.0867

H Smaoui, A Bouafsoun, A Kechrid. Microbiology, Children's Hospital of Tunis, Tunis, Tunisia

Bacterial meningitis is associated with high mortality and neurological squeal world wide.

We reported epidemiological characteristics of laboratory confirmed bacterial meningitis in children during 2000 to 2011 period.

We analyzed all laboratory confirmed bacterial meningitis cases. The serotypes and serogroups were determined by slide agglutination. Antibiotic susceptibility was determined by disk diffusion method according to CA-SFM guidelines. Beta-lactamase production was analyzed using cefinase test. MIC of beta-lactams was determined by E-test method (AB BIODISK).

During the study period we have collected 486 cases of bacterial meningitis: 157 cases of $S$. pneumoniae (32.3\%), 118 of $N$. meningitidis (24.3\%), and 99 of $H$. influenzae (20.4\%). Most cases $(66.5 \%)$ occurred in children under 3 years. The most frequent serotype among S. pneumoniae was 14 (27.2\%) followed by $23 \mathrm{~F}$ (9\%). The majority of $N$ meningitidis strains belonged to serogroup B (72.9\%) and $88.8 \%$ of $H$. influenzae strains to serotype $b$.

Before Hib conjugate vaccine introduction (2000-2002), $H$. influenzae were the first species responsible for meningitis (40\%). During vaccine generalisation (2003-2005) we noted a decrease in Hib meningitis. At the beginning of 2006, Hib vaccination was stopped and we observed an increase of Hib meningitis cases. Antimicrobial susceptibility studies show that $43.6 \%$ and $60 \%$ respectively of $S$. pneumoniae and $N$. meningitidis strains had reduced susceptibility to penicillin. Among $H$. influenzae $47.3 \%$ was betalactamase producing.

The data presented in this study demonstrate that $S$. pneumoniae is the most frequent in bacterial meningitis in children and that beta-lactams resistance is frequent in our hospital.

\section{THE PREVALENCE OF HBS ANTIGEN AND ANTI-HBS ANTIBODY AMONG PREGNANT WOMEN IN FARS PROVINCE, SOUTHERN OF IRAN}

doi:10.1136/archdischild-2012-302724.0868

1,2M Motamedifar, ${ }^{2} \mathrm{E}$ Amini, ${ }^{2} \mathrm{~J}$ Sarvari, ${ }^{2} \mathrm{P}$ Talezadeh Shirazi. 'Shiraz HIV/Aids Research Center (SHARC), Shiraz University of Medical Sciences, Iran; ${ }^{2}$ Department of Bacteriology \& Virology, Medical School, Shiraz University of Medical Sciences, Shiraz, Iran

Background and Aims Hepatitis B virus (HBV) infection is a worldwide health problem. Vertical transmission is a route of HBV infection that is regarded as a considerable factor in HBV prevention program all over the world. This study was conducted to screen the hepatitis B antigen (HBsAg) and hepatitis B surface anti body (Anti$\mathrm{HBs} \mathrm{Ab}$ ) in pregnant women in Fars province southern of Iran.

Methods From September to October 2009, two hundred fifty pregnant women in Zeynabieh hospital of Shiraz were recruited consecutively to this study at the time of their delivery. Sera were examined for the detection of $\mathrm{HBsAg}$ and Anti-HBs $\mathrm{Ab}$ using enzyme-linked immunosorbent assay (ELISA). Different demographic data (i.e. age, nationality, vaccination and family history of $\mathrm{HBV}$ infection) were investigated to determine the presence or absence of HBsAg and Anti-HBs Ab.

Results Among 250 subjects the prevalence of HBsAg and Anti$\mathrm{HBs} \mathrm{Ab}$ were $1.2 \%$ and $50 \%$ respectively. Demographic data that showed significant association with prevalence of $\mathrm{HBsAg}$ were family history of HBV infection $\left(X^{2}=7.735, p=0.05\right)$, age $\left(X^{2}=3.762\right.$, $p=0.05)$ and nationality $\left(X^{2}=5.41, p=0.02\right)$.

Conclusions Findings of this study recommend the necessity of $\mathrm{HBsAg}$ screening test for pregnant women especially for those younger than 25 years and with the family history of HBV infection and also for immigrants.

\section{CLINICS SIMILARITIES AND DIFFERENCES OF ACUTE VIRAL INFECTIONS HEPATITIS A AND E IN CHILDREN IN MONTENEGRO}

doi:10.1136/archdischild-2012-302724.0869

'D Terzic, 'B Dupanovic, 'B Andric, '1 Djecevic, ${ }^{2 Z}$ Terzic. ' ${ }^{2}$ Clinic for Infectious Diseases, ${ }^{2}$ Surgery Clinic, Clinic Center of Montenegro, Podgorica, Montenegro

The Aim To compare the clinical caracteristics of hepatitis A virus (HAV) and hepatitis $\mathrm{E}$ virus (HEV) infections in children in Montenegro. 